\title{
On the Structure of
}

\section{Asymptotically de Sitter and}

\section{Anti-de Sitter Spaces}

\author{
Michael T. Anderson \\ Department of Mathematics \\ SUNY at Stony Brook \\ Stony Brook, NY 11794-3651 \\ anderson@math.sunysb.edu
}

\begin{abstract}
We discuss several aspects of the relation between asymptotically AdS and asymptotically dS spacetimes including: the continuation between these types of spaces, the global stability of asymptotically dS spaces and the structure of limits within this class, holographic renormalization, and the maximal mass conjecture of Balasubramanian-deBoer-Minic.
\end{abstract}

\section{Introduction.}

This paper deals with several distinct issues on local and global aspects of asymptotically de Sitter spaces and their anti-de Sitter or hyperbolic counterparts. Besides their intrinsic interest in classical general relativity,

e-print archive:

http://lanl.arXiv.org/abs/hep-th/0407087 
asymptotically de Sitter spaces arise frequently in the context of inflationary models and issues related to the cosmic no-hair conjecture [1], [2]. Moreover, they are of current interest in attempts to understand a possible dS/CFT correspondence [3], [4] analogous to the much more rigorously established AdS/CFT correspondence [5]-[7].

Asymptotically de Sitter (dS) spaces are understood here to be vacuum solutions to the Einstein equations with $\Lambda>0$ which to the future (or past), have geometry asymptotically approaching that of pure de Sitter space locally. Globally, these spaces may be quite different than de Sitter; in particular future space-like infinity $\mathcal{I}^{+}$may have arbitary topology and induced metric. Asymptotically anti-de Sitter (AdS) or hyperbolic (AH) spaces are understood in the same sense; see $\S 2$ for the precise definitions.

It has long been known that there is a formal correspondence between asymptotically dS spaces and AH spaces, based on viewing each as a hyperboloid in a vacuum $(\Lambda=0)$ spacetime of one higher dimension. The continuation from $\mathrm{AH}$ to $\mathrm{dS}$ takes place across the null cone of a given point, as in the usual hyperboloidal decomposition of Minkowski space, cf. [8], and [9]-[11] for instance for more recent discussion. In $\S 2$, this formal process is made exact, and gives a rigorous form of Wick rotation or continuation between these classes of metrics; in addition, some ambiguities in the choice of "analytic continuation" are addressed.

Perhaps the most significant classical result on de Sitter (dS) spacetimes is the stability result of Friedrich [12]; in $3+1$ dimensions, the class $d S^{ \pm}$of globally hyperbolic dS spacetimes which have smooth and compact future and past conformal infinity $\mathcal{I}^{+}$and $\mathcal{I}^{-}$, is open in a natural topology. Thus, Cauchy data of any given $(M, g) \in d S^{ \pm}$may be perturbed in a small but arbitrary way, giving rise to spacetimes $(M, \widetilde{g})$ in $d S^{ \pm}$with the same overall global structure as $(M, g)$. One expects such a result is also true in higher dimensions, but a proof of this is lacking.

In $\S 3$, we describe the structure of the possible limits of spaces in $d S^{ \pm}$, i.e. elements in the boundary $\partial\left(d S^{ \pm}\right)=\overline{d S^{ \pm}} \backslash d S^{ \pm}$, at least in $3+1$ dimensions. All limits are globally hyperbolic and geodesically complete and can be one of three general types; (I) a pair of spaces $\left(M, g^{+}\right)$and $\left(M, g^{-}\right)$, infinitely far apart, and with fully degenerate $\mathcal{I}^{-}$or $\mathcal{I}^{+}$respectively, or (II) a single space $\left(M, g^{+}\right)$with partially or fully degenerate $\mathcal{I}^{-}$, or (III), a single space $\left(M, g^{-}\right)$with partially or fully degenerate $\mathcal{I}^{+}$. In particular, singularities occur only for Cauchy data "outside" the boundary $\partial\left(d S^{ \pm}\right)$. This result is also valid for all dimensions in which Friedrich's theorem holds. 
From several perspectives, the most natural limits are those of type I. As discussed in $\S 4$, such limit behavior occurs very clearly and explicitly in the family of dS Taub-NUT metrics on $\mathbb{R} \times S^{3}$, (and its higher dimensional generalizations). No examples are known where the limits are of type II or III.

In $\S 5$, we discuss holographic renormalization in the $\mathrm{dS}$ context, and relate the AH, AdS, and dS holographic stress-energy tensors and conserved quantities, cf. [13]-[15], [9], [11]. In [15], an interesting maximal mass conjecture was proposed for spaces in $d S^{+}$, in analogy to positive mass theorems in AdS and AH spaces, and also in analogy to entropy bounds in $d S^{+}$spaces, [16]. Thus, it was conjectured that any space $(M, g)$ in $d S^{+}$has holographic mass $m$ satisfying

$$
m \leq m_{0},
$$

where $m_{0}$ is the mass of pure de Sitter space (in static coordinates), unless $(M, g)$ has a cosmological singularity. In particular, any space $(M, g) \in d S^{ \pm}$ should satisfy (1.1). We refer to [17]-[20] for prior work and commentary on this conjecture.

However, we find in $\S 5$ general counterexamples to this conjecture in $3+1$ dimensions. Depending on the exact formulation of the conjecture, counterexamples are also found in $n+1$ dimensions, for all $n$ odd, and there is strong evidence that it fails also for all $n$ even.

I would like to thank V. Balasubramanian, J. de Boer and D. Minic for interesting correspondence on these issues.

\section{Asymptotic Analytic Continuation.}

Let $M$ be the interior of a compact manifold $\bar{M}$ with boundary $\partial M$. A complete Riemannian metric $g$ on $M$ is conformally compact if there is a defining function $\rho: \bar{M} \rightarrow \mathbb{R}$ such that the compactified metric

$$
\bar{g}=\rho^{2} g
$$

extends smoothly to a Riemannian metric on $\partial M$. A defining function $\rho$ satisfies $\rho \geq 0, \rho^{-1}(0)=\partial M$ and $d \rho \neq 0$ on $\partial M$. The induced metric $\gamma=\left.\bar{g}\right|_{\partial M}$ is called a boundary metric for $g$. Since defining functions are unique only up to multiplication by positive functions, only the conformal class $[\gamma]$ is an invariant of $(M, g)$ and $[\gamma]$ is called the conformal infinity of $(M, g)$. we will always assume that $M$ is connected, although the boundary $\partial M$ may be connected or disconnected. 
If $g$ is an Einstein metric on $M$, so that

$$
\text { Ric }_{g}-\frac{R}{2} g+\Lambda g=0
$$

then it is easy to see that if $\Lambda>0$, or $\Lambda=0$, then $(M, g)$ cannot be conformally compact; in the former case $\partial M=\emptyset$ while in the latter case one has at best $\partial M=\{p t\}$. Thus, $\Lambda<0$ and such metrics are called asymptotically hyperbolic (AH) or asymptotically Euclidean anti de Sitter (EAdS) Einstein metrics. As usual, define the length scale $l$ by $\Lambda=-n(n-$ 1) $/ 2 l^{2}$.

A compactification $\bar{g}=\rho^{2} g$ as in (2.1) is geodesic if $\rho(x)=\operatorname{dist}_{\bar{g}}(x, \partial M)$. Such compactifications are especially useful for computational purposes, and for the remainder of the paper we work only with geodesic compactifications. Each choice of boundary metric $\gamma \in[\gamma]$ determines a unique geodesic defining function $\rho$. If $l \neq 1$, it is convenient to work with the dimensionless function $\rho=\operatorname{dist}_{\bar{g}}(\partial M, \cdot) / l$.

The Gauss Lemma gives the splitting

$$
\bar{g}=l^{2}\left(d \rho^{2}+g_{\rho}\right), \quad g=\left(\frac{l}{\rho}\right)^{2}\left(d \rho^{2}+g_{\rho}\right),
$$

where $g_{\rho}$ is a curve of metrics on $\partial M$. In the following, it is convenient to work in the scale $l=1$, (which can always be achieved by rescaling (2.3). The Ricatti equation associated to the splitting (2.3) then states

$$
A^{\prime}+A^{2}+R_{T}=0,
$$

where $A$ is the 2 nd fundamental form of the level sets $S(\rho)$ of $\rho, A^{\prime}=\nabla_{T} A$ with $T=\nabla \rho$ and $R_{T}(V)=\langle R(T, V) V, T\rangle$; all computations here are with respect to $\bar{g}$. Using this and the Gauss equations for $S(\rho) \subset(M, \bar{g})$ together with standard formulas for the curvatures of conformally equivalent metrics, one obtains for geodesic compactifications the relation

(4) $\rho \ddot{g}_{\rho}-(n-1) \dot{g}_{\rho}-2 H g_{\rho}=\rho\left[2 R i c_{\rho}-H \dot{g}_{\rho}+\left(\dot{g}_{\rho}\right)^{2}-2\left(R i c_{g}+n|d \rho|^{2} g\right)^{T}\right]$,

where $\dot{g}_{\rho}=\mathcal{L}_{T} g_{\rho}=\frac{1}{2} A, R i c_{\rho}$ is the intrinsic Ricci curvature of the level sets $S(\rho)$ of $\rho, H=\operatorname{tr} A$ is the mean curvature of $S(\rho)$ in $(M, g)$ and $T$ denotes orthogonal projection onto the level sets $S(\rho)$.

In particular, when $g$ is Einstein, $R i c_{g}+n|d \rho|^{2} g=0$ and (2.4) gives

$$
\begin{array}{r}
\rho \ddot{g}_{\rho}-(n-1) \dot{g}_{\rho}-2 H g_{\rho}=\rho\left[2 R i c_{\rho}-H \dot{g}_{\rho}+\left(\dot{g}_{\rho}\right)^{2}\right], \\
\rho \dot{H}-H=\rho|A|^{2}, \\
\delta A=-d H,
\end{array}
$$

where the latter two equations arise from the trace of the Ricatti equation and the Gauss-Codazzi equations respectively. 
Setting $g_{0}=g_{(0)}=\gamma$, one has $g_{(1)}=\left.\dot{g}_{\rho}\right|_{\rho=0}=0$. By differentiating (2.5) with respect to $\rho$ inductively, one obtains a formal series expansion for the curve $g_{\rho}$; this is the Fefferman-Graham expansion [21]. The exact form of the expansion depends on whether $n$ is odd or even. If $n$ is odd, then

$$
g_{\rho} \sim g_{(0)}+\rho^{2} g_{(2)}+\ldots+\rho^{n-1} g_{(n-1)}+\rho^{n} g_{(n)}+\rho^{n+1} g_{(n+1)}+\ldots
$$

This expansion is even in powers of $\rho$ up to order $n-1$. The coefficients $g_{(2 k)}, k \leq(n-1) / 2$ are locally determined by the boundary metric $\gamma=g_{(0)}$; they are explicitly computable expressions in the curvature of $\gamma$ and its covariant derivatives. The last two equations in (2.5) imply that the term $g_{(n)}$ is transverse-traceless, i.e.

$$
t r_{\gamma} g_{(n)}=0, \quad \delta_{\gamma} g_{(n)}=0,
$$

but $g_{(n)}$ is otherwise undetermined by $\gamma$; it depends on the global structure of the AH Einstein metric $(M, g)$. For $k>n$, terms $g_{(k)}$ occur for both $k$ even and odd; the term $g_{(k)}$ depends on two boundary derivatives of $g_{(k-2)}$.

If $n$ is even, one has

$$
g_{\rho} \sim g_{(0)}+\rho^{2} g_{(2)}+\ldots .+\rho^{n-2} g_{(n-2)}+\rho^{n} g_{(n)}+\rho^{n} \log \rho h_{(n)}+\ldots
$$

Again the terms $g_{(2 k)}$ up to order $n-2$ are explicitly computable from the boundary metric $\gamma$, as is the transverse-traceless coefficient $h_{(n)}$ of the first $\log \rho$ term. The term $h_{(n)}$ is an important term for the corresponding CFT on $\partial M$; up to a constant, it is the metric variation of the conformal anomaly, cf. [14], (or also [22]). The term $g_{(n)}$ satisfies

$$
\operatorname{tr}_{\gamma} g_{(n)}=\tau, \quad \delta_{\gamma} g_{(n)}=\delta,
$$

where $\tau$ and $\delta$ are explicitly determined by the boundary metric $\gamma$ and its derivatives, but again $g_{(n)}$ is otherwise undetermined by $\gamma$. The series (2.8) is even in powers of $\rho$, and terms of the form $\rho^{k}(\log \rho)^{l}$ appear at order $k>n$. Again the coefficients $g_{(k)}$ and $h_{(k)}$ depend on two derivatives of $g_{(k-2)}$ and $h_{(k-2)}$. The expansions (2.6) and (2.8) of course depend on the choice of boundary metric $\gamma \in[\gamma]$. However, transformation properties of the coefficients $g_{(n)}$ and $h_{(n)}$ under conformal changes are readily computable, cf. [14], [23].

Mathematically, the expansions (2.6) and (2.8) are formal, obtained by compactifiying the Einstein equations and taking iterated Lie derivatives of $\bar{g}$ at $\rho=0$. If $\bar{g} \in C^{m, \alpha}(\bar{M})$, then the expansions hold up to order $m+\alpha$. However, boundary regularity results are needed to ensure that if an $\mathrm{AH}$ Einstein metric $g$ with boundary metric $\gamma$ satisfies $\gamma \in C^{m, \alpha}(\partial M)$, then the compactification $\bar{g} \in C^{m, \alpha}(\bar{M})$. These have recently been established in general in [24] for $n=3$, and in [25] for $n \geq 3$ in case of $C^{\infty}$ boundary metric $\gamma$. 
In sum, an AH Einstein metric is formally determined near $\partial M$ by $g_{(0)}$ and $g_{(n)}$. The term $g_{(0)}$ corresponds to Dirichlet boundary data on $\partial M$, while $g_{(n)}$ corresponds to Neumann boundary data, (in analogy with the scalar Laplace operator). Thus, for global AH Einstein metrics defined on a compact manifold with boundary $\bar{M}=M \cup \partial M$, the correspondence

$$
g_{(0)} \rightarrow g_{(n)}
$$

is analogous to the Dirichlet-to-Neumann map for harmonic functions. As discussed in $\S 5$, the term $g_{(n)}$ is essentially the (renormalized) Brown-York quasilocal stress-energy tensor of $(M, g)$ and corresponds to the expectation value of the stress-energy tensor of the dual CFT on $\partial M$ in all known cases. However, the map (2.10) per-se is only well-defined if there is a unique $\mathrm{AH}$ Einstein metric with boundary data $\gamma=g_{(0)}$. This occurs in some situations, but fails in others.

On the other hand, the expansions (2.6) and (2.8) are completely local, both in the distance to the boundary $\rho$, and tangentially on $\partial M$. If one restricts only to local considerations, then the free data in the expansion are $g_{(0)}$ and $g_{(n)}$, subject to the constraint equations (2.7) and (2.9). Of course arbitrarily given $g_{(0)}$ and $g_{(n)}$ will not correspond to globally smooth solutions on a compact manifold $\bar{M}$.

Next we turn to Lorentzian metrics. The definition of a conformal completion for Lorentz metrics is similar, although more subtle, than the Riemannian case due to the causal structure and the common occurence of singularities. Note however that for local considerations, such as the computation of the coefficients $g_{(k)}$ in the expansions (2.6) or (2.8), global issues are irrelevant.

As is well-known, the structure of the boundary of a conformal completion depends on the sign of $\Lambda$. If $\Lambda<0$, the AdS case, then $\partial M=\mathbb{R} \times \partial \Sigma$, with $\gamma$ a Lorentz metric. For simplicity, we will always assume that $\partial \Sigma$ is compact and, abusing notation slightly, call $(M, g)$ conformally compact if the definition (2.1) holds with $\partial M$ of the form above. Thus, $\partial \Sigma$ is the conformal boundary of a space-like slice $\Sigma$ in $(M, g)$, while $\partial M=\mathcal{I}$ is timelike. Note that $\mathcal{I}$ is not necessarily connected, as is the case for instance for the AdS Schwarzschild metric.

If $\Lambda>0$, the dS case, then $(\partial M, \gamma)$ is a Riemannian manifold, representing the spatial behavior at future or past infinite times. Thus, $\partial M$ may have two space-like components $\mathcal{I}^{+}, \mathcal{I}^{-}$representing future and past conformal infinity respectively, or possibly only one component, $\mathcal{I}^{+}$or $\mathcal{I}^{-}$. The definition (2.1) holds without change in this situation. However, the compactness of $\partial M$ precludes the existence of (most) singularities in $(M, g)$, and so many 
important solutions are not conformally compact. We will say that $(M, g)$ is partially conformally compact if $\partial M$ is open and non-empty. Note that $\gamma \in[\gamma]$ may then either be complete or incomplete.

If $\Lambda=0$, the flat or Minkowski case, then $(\partial M, \gamma)$ is null (degenerate), of the form $\partial M=\mathbb{R} \times \partial \Sigma$. Usually $\partial \Sigma$ is taken to be compact, and represents the boundary at infinity of a null-hypersurface in $(M, g)$. The $\mathbb{R}$ factor parametrizes null geodesics, and again $\partial M$ may have two components, $\mathcal{I}^{+}, \mathcal{I}^{-}$.

Consider now the expansions (2.6) or (2.8) in these situations.

I. Suppose $\Lambda<0$, the AdS case. Then $(M, g)$ is spatially non-compact and conformal infinity $(\partial M, \gamma)$ is Lorentzian; the vector field $\partial_{\rho}=\nabla \rho$ is space-like. All of the discussion (2.3)-(2.9) above holds without change in this setting; the only difference is that $g_{\rho}$ is curve of Lorentz metrics on $S(\rho)$.

II. Suppose $\Lambda>0$, the dS case. Then conformal infinity $(\partial M, \gamma)$ has components $\mathcal{I}^{+}, \mathcal{I}^{-}$or both. There is no geodesic defining function $\rho$ for both $\mathcal{I}^{+}$and $\mathcal{I}^{-}$simultaneously; instead one has $\rho=\rho^{+}$or $\rho=\rho^{-}$. In both situations, $\partial_{\rho}$ is time-like, and $\partial_{\rho}=-\nabla \rho$. Thus $\partial_{\rho}$ is past directed near $\mathcal{I}^{+}$ and future directed near $\mathcal{I}^{-}$.

Since $|d \rho|^{2}=-1$, (or $\left.-l^{-2}\right),(2.5)$ changes to its negative, i.e.

$$
\rho \ddot{g}_{\rho}-(n-1) \dot{g}_{\rho}-2 H g_{\rho}=-\rho\left[2 R i c_{\rho}-H \dot{g}_{\rho}+\left(\dot{g}_{\rho}\right)^{2}\right] .
$$

This leads to an expansion of the form (2.6) or (2.8) exactly as in the AH or EAdS case; for $n=3$ this expansion is due to Starobinsky [26], predating the work of Fefferman-Graham. To compare these expansions, suppose

$$
g_{(0)}^{d S}=g_{(0)}^{A H} .
$$

Then (2.5) and (2.12) immediately give $g_{(1)}^{d S}=g_{(1)}^{A H}=0$, while $g_{(2)}^{d S}=-g_{(2)}^{A H}$, (when $n>2$ ). Similarly, for $2 k<n$,

$$
g_{(2 k)}^{d S}=(-1)^{k} g_{(2 k)}^{A H} .
$$

Recall that the transverse-traceless part of the term $g_{(n)}$ is undetermined; this occurs on both the AH and dS sides. The natural relation between $g_{(n)}^{A H}$ and $g_{(n)}^{d S}$ is to set

$$
g_{(n)}^{d S}= \pm g_{(n)}^{A H} .
$$

However, there appears to be no compelling reason to choose one sign over the other. The continuation of pure hyperbolic space to pure de Sitter space, 
(in static coordinates), requires

$$
g_{(2)}^{d S}=-g_{(2)}^{A H}, \text { for } n=2 \text {, and } g_{(4)}^{d S}=g_{(4)}^{A H}, \text { for } n=4,
$$

but imposes no restrictions for other $n$. Based on the examples discussed in $\S 4$, we choose

$$
g_{(n)}^{d S}= \pm g_{(n)}^{A H},
$$

where + is chosen if $n \equiv 0,1(\bmod 4)$, while - is chosen if $n \equiv 2,3(\bmod 4)$; see also Remark 2.2 for the opposite choice. The coefficients of the AH and dS expansions are then related by

$$
g_{(k)}^{d S}= \pm g_{(k)}^{A H} \text { and } h_{(n)}^{d S}=(-1)^{n / 2} h_{(n)}^{A H},
$$

for $k>n$, where again + occurs if $k \equiv 0,1(\bmod 4)$, while - occurs if $k \equiv 2,3(\bmod 4) ;$ see the Appendix for further details.

Thus, one has a formal correspondence between AH and dS solutions of the Einstein equations, given by

$$
g^{A H}=\left(\frac{l}{\rho}\right)^{2}\left(d \rho^{2}+g_{\rho}^{A H}\right) \Longleftrightarrow g^{d S}=\left(\frac{l}{\rho}\right)^{2}\left(-d \rho^{2}+g_{\rho}^{d S}\right),
$$

where the formal expansions of $g_{\rho}^{A H}$ and $g_{\rho}^{d S}$ have coefficients related by (2.17). The metrics $g^{A H}$ and $g^{d S}$ can be formally obtained from each other by changing $\rho$ to $i \rho$ and $l$ to $i l$ in $g^{A H}$, and dropping all resulting $i$ coefficients (at odd powers of $\rho$ ) in the expansions for $g_{\rho}^{d S}$. Note that a given AH metric generates a de Sitter metric $g^{d S}$ with either a future conformal infinity $\mathcal{I}^{+}$ or a past conformal infinity $\mathcal{I}^{-}$, but not necessarily both simultaneously.

To actually construct or prove the existence of metrics from this correspondence, one needs the formal expansions to converge to $g_{\rho}$.

Theorem 2.1. There is a 1-1 correspondence between $C^{\omega}$ conformally compact Riemannian AH Einstein metrics with boundary data $\left(\gamma, g_{(n)}\right)$, and $C^{\omega}$ conformally compact Lorentzian $d S$ Einstein metrics with past (or future) boundary data $\left(\gamma, g_{(n)}\right)$, given by (2.18). Thus, given any real-analytic metric $\gamma$ and any real-analytic symmetric bilinear form $g_{(n)}$ on an $n$-manifold $\partial M$, satisfying the constraint conditions (2.7) or (2.9), there exist unique $A H$ Einstein and $d S$ Einstein metrics with boundary data $\left(\gamma, g_{(n)}\right)$, defined in a thickening of $\partial M$, and related by the correspondence (2.18).

Proof: When $n=3$, the existence and convergence of the expansions on the $\mathrm{AH}$ and dS sides has been proved in [24], and the discussion above then gives the correspondence (2.18). For general $n$, the existence and convergence of the expansions has recently been proved in [27] and [28]. 
This correspondence thus gives a rigorous form of "Wick rotation" between these types of Einstein metrics. The construction can be realized geometrically by embedding the spaces $\left(M, g^{A H}\right)$ and $\left(M, g^{d S}\right)$ into a selfsimilar vacuum spacetime $(\Lambda=0)$ of one higher dimension, exactly as the embeddings of the hyperbolic and de Sitter metrics as hyperboloids in flat Minkowski space. The transition from AH geometry to dS geometry takes place across the future or the past light cone of a fixed point.

However, this construction is not, strictly speaking, an analytic continuation of AH metrics to dS metrics. When $n$ is odd, the compactified metrics $g^{A H}$ and $g^{d S}$ are only $C^{n / 2}$ across conformal infinity, in the generic case when $g_{(n)} \neq 0$. If $n$ is even and the conformal anomaly stress-energy $h_{(n)}$ is nonvanishing, the compactifcations $g^{A H}$ and $g^{d S}$ are $C^{n / 2-\varepsilon}$ across conformal infinity.

The Einstein equations on the AH side form an elliptic system of PDE's, in a suitable gauge. Thus, at least formally, one has a well-defined Dirichlet problem at infinity. For global solutions defined on a compact manifold with boundary $\bar{M}=M \cup \partial M$, the stress-energy term $g_{(n)}$ is thus determined (formally) by the boundary metric $\gamma=g_{(0)}$. On the dS side, the Einstein equations form, again in suitable gauge, a hyperbolic system of PDE. Here the terms $g_{(0)}$ and $g_{(n)}$ form initial or Cauchy data for the evolution equations. Thus, they are freely prescribed, subject to the constraints (2.7) or (2.9) and independent of each other; of course arbitrary data $g_{(0)}, g_{(n)}$ may not give rise to global solutions.

This distinction is reflected in the behavior of symmetries of the boundary metrics at conformal infinity. If $G$ is a connected group of conformal isometries of $(\partial M,[\gamma])$, then $G$ extends to a group of isometrics of any $\mathrm{AH}$ Einstein filling metric $(M, g)$ with boundary data $(\partial M,[\gamma])$, cf. [29]. This is of course not the case on the dS side. Symmetries of $\gamma$ do not necessarily extend to symmetries of $g^{d S}$; only isometry groups preserving both $\gamma$ and $g_{(n)}$ extend to isometries of $g^{d S}$.

Remark 2.2. As noted above in the proof of Theorem 2.1, when $n$ is odd and $\gamma$ is real-analytic, the series (2.6) converges. Hence, one can continue an AH Einstein metric $(M, g)$ to the region $\rho<0$, obtaining an AH Einstein metric $\widetilde{g}$ "on the other side" of $\partial M$. One then has

$$
\widetilde{g}_{(0)}=g_{(0)} \text { and } \tilde{g}_{(n)}=-g_{(n)} \text {. }
$$

For example, when $n=3$, and for $\rho>0$, the expansion for $\widetilde{g}$ has the form

$$
\widetilde{g}_{\rho}=g_{(0)}+\rho^{2} g_{(2)}-\rho^{3} g_{(3)}+\rho^{4} g_{(4)}-\rho^{5} g_{(5)}+\ldots
$$


with $\rho>0$. This has the $\mathrm{dS}$ continuation

$$
\widetilde{g}_{\rho}^{d S}=g_{(0)}-\rho^{2} g_{(2)}+\rho^{3} g_{(3)}+\rho^{4} g_{(4)}-\rho^{5} g_{(5)}+\ldots
$$

Here, and for all $n$ odd, one thus has the opposite signs from (2.17), for $k$ odd; the series for $\widetilde{g}_{\rho}^{d S}$ is obtained from that of $g_{\rho}^{A H}$ by replacing $\rho$ by $-i \rho$, (instead of $\rho \rightarrow i \rho$ ).

However, in all known examples, if $(M, g)$ is a globally smooth AH Einstein metric, the continuation $\widetilde{g}$ has singularities; for example the positive mass AH Schwarzschild metrics continue in $\rho<0$ to the corresponding negative mass AH Schwarzschild metrics, cf. also [30] for other examples.

When $n$ is even, the term $\log \rho$ cannot be continued to $\rho<0$, and so one does not obtain an analogous $\widetilde{g}$ in this case. This also suggests that (2.16) is the right sign choice for $n$ even.

Remark 2.3. Theorem 2.1 also holds for the continuation of AdS, (i.e Lorentzian) Einstein metrics. However, the continuation is then a solution of the Einstein equations with $\Lambda>0$, and with signature $(2, n-1)$, i.e. $(--++\ldots+)$. In some circumstances, this can be Wick rotated to a Lorentzian metric, just as an AdS metric can sometimes be Wick rotated to an $\mathrm{AH}$ metric.

Remark 2.4. The analogue of the expansion (2.6) or (2.8) when $\Lambda=0$ is much more complicated, and goes back to work of Bondi and Sachs; cf. [31] for a recent discussion.

\section{$3 \quad$ Global structure of dS spaces.}

Let $d S^{+}$be the space of de Sitter spacetimes, i.e. vacuum solutions of the Einstein equations with $\Lambda>0$, which are conformally compact to the future; thus $\left(\mathcal{I}^{+}, \gamma^{+}\right)$is a compact Riemannian manifold, without boundary, with $\gamma^{+} \in\left[\gamma^{+}\right]$. The same definition holds for $d S^{-}$. Let $d S^{ \pm}$be the space of de Sitter spacetimes which are globally conformally compact. If $(M, g) \in d S^{ \pm}$ is globally hyperbolic, then it is easy to see that $M$ is geodesically complete and topologically a product of the form $M=\mathbb{R} \times \Sigma$, with Cauchy surface $\Sigma$ diffeomorphic to $\mathcal{I}^{+}$and $\mathcal{I}^{-}$. It will always be assumed that spaces in $d S^{ \pm}$ are globally hyperbolic.

Remark 3.1. A spacetime $(M, g)$ in $d S^{+} \cap d S^{-}$is necessarily globally hyperbolic in a neighborhood of $\mathcal{I}^{+}$and $\mathcal{I}^{-}$, and it is natural to conjecture that all of $(M, g)$ is globally hyperbolic. However, this is an open problem. Apriori, there may be singularities sandwiched between the Cauchy surfaces 
near $\mathcal{I}^{+}$and $\mathcal{I}^{-}$which don't propagate to either $\mathcal{I}^{+}$or $\mathcal{I}^{-}$. Although physically unlikely to occur, mathematically such solutions have not been ruled out, cf. [32] for further discussion.

For $(M, g) \in d S^{+}$, one has the data at infinity $\left(g_{(0)}^{+}, g_{(n)}^{+}\right)$on $\mathcal{I}^{+}$, from the Fefferman-Graham or Starobinsky asymptotic expansion. At least for analytic data, and up to a natural equivalence, these uniquely determine, up to isometry, the maximal globally hyperbolic solution $(M, g)$. Thus, let

$$
\mathcal{C}^{+} \subset \operatorname{Met}\left(\mathcal{I}^{+}\right) \times S^{2}\left(\mathcal{I}^{+}\right)
$$

be the subset consisting of pairs $\left(\gamma, g_{(n)}\right)$ such that $(2.7)$ or $(2.9)$ holds, depending on whether $n$ is odd or even. Let $\mathcal{A}^{+}=\mathcal{C}^{+} / \sim$, where $\gamma_{1} \sim \gamma_{2}$ if there exists $\sigma: \mathcal{I}^{+} \rightarrow \mathbb{R}$ such that $\gamma_{2}=e^{2 \sigma} \gamma_{1}$, and $\left(g_{1}\right)_{(n)} \sim\left(g_{2}\right)_{(n)}$ if $\left(g_{2}\right)_{(n)}=e^{-n \sigma}\left(g_{1}\right)_{(n)}$ when $n$ is odd; see [14], [23] for the more complicated relation on $g_{(n)}$ if $n$ is even.

Then in any dimension, any real-analytic data $\left(\gamma, g_{(n)}\right) \in \mathcal{A}^{+}$determine a unique solution up to isometry in $d S^{+}$, by [27] or [28]. When $n=3$, so that the spacetime is 4-dimensional, a result of Friedrich [12] shows that $C^{k}$ data in $\mathcal{A}^{+}$determine a unique solution in $d S^{+}$, for $k \geq 7$ for instance. Of course the same results hold with $\mathcal{C}^{-}$and $d S^{-}$.

When $n=3$, a basic result of Friedrich [12] is that $d S^{ \pm}$is open in the $C^{k}$ topology, i.e. given any solution $\left(M, g_{0}\right) \in d S^{ \pm}$, any sufficiently small perturbation of the initial data in $\mathcal{A}^{-}$at $\mathcal{I}^{-}$, (or of the final data in $\mathcal{A}^{+}$at $\left.\mathcal{I}^{+}\right)$gives rise to a global solution $(M, g)$ in $d S^{ \pm}$near $\left(M, g_{0}\right)$. This applies in particular to de Sitter space itself, so any small perturbation of the pure dS data $\left(\gamma_{S^{3}(1)}, 0\right)$ on $\mathcal{I}^{+}$or $\mathcal{I}^{-}$gives rise to a complete solution $(M, g)$ in $d S^{ \pm}$.

One certainly expects Friedrich's theorem to be true in all dimensions, but a rigorous mathematical proof of this is lacking. It would suffice to prove a Cauchy stability theorem for the degenerate (Fuchsian) system of PDE obtained by conformally compactifying the Einstein equations, as in (2.5). For analytic boundary data say at $\mathcal{I}^{+}$, Fuchsian versions of the CauchyKovalevsky theorem give the existence of the analytic or polyhomogenous solutions $(M, g) \in d S^{+}$mentioned above. However, it is well-known that in general, solutions given by the Cauchy-Kovalevsky theorem do not necessarily vary continuously with the initial data.

In the opposite direction, there are two general results, valid in all dimensions, restricting the boundary data on $\mathcal{I}^{+}$or $\mathcal{I}^{-}$of metrics in $d S^{ \pm}$. First, it is proved in [33], [34] that if $(M, g) \in d S^{+}$has a representative 
metric $\gamma^{+} \in\left[\gamma^{+}\right]$of negative scalar curvature, $R_{\gamma^{+}}<0$, (or $R_{\gamma^{+}} \leq 0$ ), then $\mathcal{I}^{-}=\emptyset$, so that there is not even a partial compactification at past infinity. In fact if $\gamma^{+}$is chosen to have constant negative scalar curvature $R<0$, there is an upper bound $\rho_{0}$ on the distance to $\mathcal{I}^{+}$in the geodesic compactification,

$$
\rho \leq \rho_{0}=\frac{4 n(n-1)}{|R|}
$$

Second, the following interesting result has been proved in [33]; if $(M, g) \in$ $d S^{+}$and $\left|\pi_{1}\left(\mathcal{I}^{+}\right)\right|=\infty$, then again $\mathcal{I}^{-}=\emptyset$. When $n=3$, the only known 3 -manifolds with finite fundamental group are spherical space-forms $S^{3} / \Gamma$. Perelman's work [35] implies that in fact these are the only such 3-manifolds. Up to finite covering projections, it follows that one must have $\mathcal{I}^{+}=S^{3}$ and $M=\mathbb{R} \times S^{3}$ for spaces in $d S^{ \pm}$, regardless of the data in $\mathcal{C}^{+}$.

Consider the (gravitational) scattering map

$$
\begin{gathered}
\mathcal{S}: \mathcal{A}^{-} \rightarrow \mathcal{A}^{+}, \\
\mathcal{S}\left[\gamma^{-}, g_{(n)}^{-}\right]=\left[\gamma^{+}, g_{(n)}^{+}\right] .
\end{gathered}
$$

Here $g_{(n)}^{ \pm}$are taken with respect to the future normal direction; hence $g_{(n)}^{+}$ differs in sign from the term $g_{(n)}$ in $(2.6)$ or $(2.8)$, since at $\mathcal{I}^{+}, \partial_{\rho}$ is past directed.

When $n=3$, using either the linearized conformal field equations [12] or the linearized Bach equations, cf. [25], on the compactified (unphysical) metric $\bar{g}$, it is straightforward to prove that $D_{g} \mathcal{S}$ is an isomorphism, for any $g \in d S^{ \pm}$. This is because the linearized equations at $\bar{g}$ are a linear system of wave equations, (in a suitable gauge), on a compact manifold with finite time interval. Again, one expects such a result to be true in all dimensions, but this remains to be proved.

For $n=3$, it follows that one may locally parametrize $d S^{ \pm}$by conformal classes of metrics $\left(\gamma^{-}, \gamma^{+}\right)$on $\mathcal{I}^{-} \times \mathcal{I}^{+}$instead of $\mathcal{A}^{-}$or $\mathcal{A}^{+}$. Further, $\left\|D \mathcal{S}_{g}\right\|$ is bounded, with a bound depending on $g$. However, as will be seen below and in $\S 4,\left\|D_{g} \mathcal{S}\right\|$ blows up on bounded sequences of data in $\mathcal{A}^{-}$or $\mathcal{A}^{+}$, or $\mathcal{I}^{-} \times \mathcal{I}^{+}$.

It is of interest to examine the structure of the closure of the open set $d S^{ \pm}$. What kind of spaces lie in $\partial\left(d S^{ \pm}\right)=\overline{d S^{ \pm}} \backslash d S^{ \pm}$, where the closure is taken in the smooth topology induced via (3.1)? Recall that solutions in $d S^{ \pm}$are conformally compact, and so conformally equivalent to a smooth bounded metric on the product $I \times \Sigma$. Generically, such metrics are "tall" in the sense of [36], [37], in that they contain Cauchy surfaces entirely visible 
to observers at sufficiently late times. A spacetime $(M, g) \in d S^{+}$(or $d S^{-}$) is called "infinitely tall" if it is conformally equivalent to a complete, (but not necessarily product), metric on $[0, \infty) \times \Sigma$ with $\{0\} \times \Sigma=\mathcal{I}^{+}$, (or $\mathcal{I}^{-}$).

Theorem 3.2. An element in the boundary $\partial d S^{ \pm}$of $d S^{ \pm}$, for $n=3$, is given by exactly one of the following three configurations:

I. A pair of solutions $\left(M, g^{+}\right) \in d S^{+}$and $\left(M, g^{-}\right) \in d S^{-}$, each geodesically complete and globally hyperbolic. One has $\mathcal{I}^{-}=\emptyset$ for $\left(M, g^{+}\right)$and $\mathcal{I}^{+}=\emptyset$ for $\left(M, g^{-}\right)$. Both solutions $\left(M, g^{+}\right)$and $\left(M, g^{-}\right)$are infinitely tall, and "infinitely far apart".

II. A single geodesically complete and globally hyperbolic solution $(M, g) \in$ $d S^{+}$, either with a partial compactification at $\mathcal{I}^{-}$, or $\mathcal{I}^{-}=\emptyset$.

III. A single geodesically complete and globally hyperbolic solution $(M, g) \in$ $d S^{-}$, either with a partial compactification at $\mathcal{I}^{+}$, or $\mathcal{I}^{+}=\emptyset$.

Proof: One can work at either $\mathcal{I}^{+}$or $\mathcal{I}^{-}$, and so we choose $\mathcal{I}^{+}$. Given data $\left(g_{(0)}^{+, i}, g_{(3)}^{+, i}\right) \in \mathcal{C}^{+}$giving solutions $\left(M, g^{i}\right)$ in $d S^{ \pm}$, suppose $g_{(0)}^{+, i} \rightarrow g_{(0)}^{+}$, and $g_{(3)}^{+, i} \rightarrow g_{(3)}^{+}$in the $C^{k},\left(k \geq 7\right.$ for instance), topology on $\mathcal{C}^{+}$, (or analytic topology for analytic data). The induced data on $\mathcal{I}^{-}$may either converge to a limit, (in a subsequence), or diverge to infinity in $\mathcal{C}^{-}$.

Suppose first the data on $\mathcal{I}^{-}$converge to a limit, so that

$$
g_{(0)}^{-, i} \rightarrow g_{(0)}^{-}, \text {and } g_{(3)}^{-, i} \rightarrow g_{(3)}^{-} \text {. }
$$

Of course, by definition, the metrics $\left(M, g^{i}\right)$ do not converge to a limit $(M, g) \in d S^{ \pm}$.

By Friedrich's theorem [12], there exist maximal globally hyperbolic $d S^{+}$ and $d S^{-}$solutions $\left(M, g^{+}\right)$and $\left(M, g^{-}\right)$, defined at least for $(T, \infty)$ and $(-\infty,-T)$ respectively, which realize the limit boundary data at $\mathcal{I}^{+}$and $\mathcal{I}^{-}$. Here $T=-\log \rho_{0}$, where $\rho^{ \pm}$is the geodesic defining function for $\mathcal{I}^{+}$or $\mathcal{I}^{-}$, and $\rho^{ \pm}=\rho_{0}$ is small. Further, on $\left(\rho^{+}\right)^{-1}(T, \infty)$ and $\left(\rho^{-}\right)^{-1}(-\infty,-T)$, the metrics $g^{i} \rightarrow g^{+}$and $g^{i} \rightarrow g^{-}$respectively. (This fact is the only place in the proof where the condition $n=3$ is needed).

Let $S_{i}\left(T^{+}\right)$be the $T^{+}$level set of $t^{+}=-\log \rho^{+}$with respect to $g^{i}$ and similarly $S_{i}\left(T^{-}\right)$the $T^{-}$level set of $t^{-}=\log \rho^{-}$. We claim that the geodesic distance between $S_{i}\left(T^{-}\right)$and $S_{i}\left(T^{+}\right)$diverges to $\infty$, as $i \rightarrow \infty$, i.e. all timelike maximal geodesics joining $S_{i}\left(T^{-}\right)$to $S_{i}\left(T^{+}\right)$have length diverging to $\infty$ as $i \rightarrow \infty$. To see this, the Cauchy data of $g^{i}$ at $S_{i}\left(T^{-}\right)$and $S_{i}\left(T^{+}\right)$ converge to the Cauchy data of $g^{-}$at $S\left(T^{-}\right)$and the Cauchy data of $g^{+}$ 
at $S\left(T^{+}\right)$respectively. Since the solutions $g^{i}$ exist from time $T^{-}$to $T^{+}$, (and much further), the Cauchy stability theorem for the vacuum Einstein equations implies that the limit solution $g$ exists between $S\left(T^{-}\right)$and $S\left(T^{+}\right)$ if the distance between $S_{i}\left(T^{-}\right)$and $S_{i}\left(T^{+}\right)$remains uniformly bounded, (at all points). Hence, $g^{+}$and $g^{-}$are part of a global solution $(M, g) \in d S^{ \pm}$, giving a contradiction. It follows that the limit solutions $g^{+}$and $g^{-}$are infinitely far apart.

Exactly the same reasoning, choosing suitable $T^{-}$or $T^{+}$, proves that each maximal globally hyperbolic spacetime $\left(M, g^{+}\right)$and $\left(M, g^{-}\right)$is geodesically complete.

Next, we need to show that $g^{+}$has $\mathcal{I}^{-}=\emptyset$. (The argument that $\mathcal{I}^{+}=\emptyset$ for $g^{-}$is the same). This will also prove that $g^{+}$is infinitely tall. Suppose instead $\mathcal{I}^{-} \neq \emptyset$, so that there is a least a partial compactification $\mathcal{I}^{-}$of $g^{+}$ at past infinity.

For $i$ sufficiently large, the metrics $g^{i}$ are (arbitrarily) close to the limit $g^{+}$on (arbitrarily) large regions of $g^{+}$. Of course, by the analysis above, the metrics $g^{i}$ extend "much further" to the past of $g^{+}$. Fix $i$ large for the moment, and let $\rho=\rho_{i}^{-}$be the geodesic defining function for past infinity $\left(\mathcal{I}^{-}, g_{(0)}^{-, i}\right)$ of $\left(M, g^{i}\right)$. Let $H$ be the mean curvature of the level sets of $\rho$ with respect to $g^{i}$. By [34], the ratio

$$
\frac{3-H}{\rho^{2}}=\frac{\bar{H}}{\rho}
$$

is monotone decreasing in $\rho$. Here $\bar{H}$ is the mean curvature of the same level sets with respect to the compactified metric $\bar{g}=\bar{g}^{i}$ as in (2.1);H is taken with respect to the future, while $\bar{H}$ is taken with respect to the past unit normal, so $H>0$ corresponds to expansion to the future. Let $S(\rho)$ denote the levels sets of $\rho$.

Now choose $T=T_{i}$ large enough so that $S(T) \subset\left(M, g_{i}\right)$ is partially close to a domain in $g^{+}$near past infinity $\mathcal{I}^{-}$in $g^{+}$. Thus there is a domain $U \subset S(T) \subset\left(M, g^{i}\right)$ such that $\left(U, \bar{g}^{+}\right)$is close to a domain $U_{\infty} \subset\left(\mathcal{I}^{-}, g_{(0)}^{-}\right)$. Since $\rho$ is extremely large on $S(T)$, the ratio in (3.5) is very small. However, (3.5) holds for any geodesic compactification and one can renormalize $\rho$ by sending $\rho \rightarrow \varepsilon \rho=\widetilde{\rho}$. This change of compactification makes the metric $g_{(0)}^{-, i}$ on $\mathcal{I}^{-}$very small; $\widetilde{g}_{(0)}^{-, i}=\varepsilon^{2} g_{(0)}^{-, i}$. Setting $\varepsilon=T^{-1}$ then gives $\widetilde{\rho}=1$ on $S(T)$. The numerator $3-H$ in (3.5) is of course "scale-invariant"; it does not depend on scale of $\rho$. 
It follows now from the choice of $T$ that the ratio

$$
\frac{3-H}{\widetilde{\rho}^{2}}
$$

in (3.5) is very small; it can be made arbitrarily small by choosing $i$ sufficiently large and $T$ sufficiently large so that $U \subset S(T)$ is sufficiently close to $U_{\infty}$. Now the monotonicity of (3.5), (applied on each $g^{i}$ ), implies that as $\widetilde{\rho}$ increases, the ratio (3.6) becomes even smaller.

It follows that in the limit, the domain in $g^{+}$formed by $\rho$-curves between $\mathcal{I}^{-}$and $\mathcal{I}^{+}$has $H \equiv(n-1)$. Via standard arguments with the Raychaudhuri equation, it follows that this region is isometric to pure de Sitter space. Since $g^{+}$is globally hyperbolic and geodesically complete, it then follows that $g^{+}$ is globally isometric to pure de Sitter space. Hence, the limit data $\left(g_{(0)}^{+}, g_{(3)}^{+}\right)$ equals the data $\left(\gamma_{0}, 0\right)$ of pure de Sitter space, a contradiction.

Next suppose (3.4) fails, so that either $g_{(0)}^{i,-}$ or $g_{(3)}^{i,-}$ diverges to $\infty$ on $\mathcal{I}^{-}$. The same reasoning as before shows that the limit $(M, g) \in d S^{+}$is geodesically complete and globally hyperbolic. In this setting, one then has only a partial past infinity $\mathcal{I}^{-}$, given by the domain (possibly empty) on which both $g_{(0)}^{i,-}$ and $g_{(3)}^{i,-}$ converge to the limit $\left(g_{(0)}^{-}, g_{(3)}^{-}\right)$. This situation then gives Case II. Case III is obtained in the same way, by interchanging $\mathcal{I}^{-}$with $\mathcal{I}^{+}$.

Exactly the same proof holds in all dimensions if an openness or Cauchy stability result holds, as in the case $n=3$.

Examples discussed in $\S 4$, (the Taub-NUT curve) show that the configuration in Case I does occur. On the other hand, there are no known examples where the configuration in Case II or III above occurs, and it would be interesting to know if such configurations can actually arise or not.

It would also be interesting to prove that the completion $\overline{d S^{ \pm}}$is compact in the topology on $\mathcal{A}^{+}$for instance, i.e. if the final data $\left(\gamma^{+}, g_{(n)}^{+}\right)$on $\mathcal{I}^{+}$ are sufficiently large, then the corresponding solution cannot be in $d S^{ \pm}$. The condition that $(M, g) \in d S^{ \pm}$implies $R_{\gamma^{+}}>0$, (and $R_{\gamma^{-}}>0$ ), is one measure of this. Finally, one would like to know if the space $d S^{ \pm}$is connected, as a domain in $\mathcal{A}^{+}$for instance.

These questions correspond to the picture that $d S^{ \pm}$is given by a connected, bounded domain in $\mathcal{A}^{+}$, (or $\mathcal{A}^{-}$), with a wall or boundary described by Theorem 3.1. For initial data on $\mathcal{A}^{-}$for instance, one expects the formation of black holes and big bang type singularities outside the wall. 
Finally, consider limits $(M, g) \in d S^{+}$of $d S^{ \pm}$which have $\mathcal{I}^{-}=\emptyset$. For simplicity, assume $n=3$, so that $M$ is then topologically of the form $\mathbb{R} \times S^{3}$, (in a finite cover). Thus, as $T \rightarrow-\infty$, the Cauchy surfaces $S(T)$ degenerate when compactified. The simplest example of a diverging curve metrics on $S^{3}$ is a collapsing family, where the lengths of the circle fibers $S^{1}$ in the Hopf fibration $S^{3} \rightarrow S^{2}$ have length converging to 0. This type of degeneration occurs with uniformly bounded curvature tensor, and is illustrated concretely on the Taub-NUT curve, discussed below.

\section{Examples.}

In this section, we discuss several classes of examples illlustrating the work in $\S 3$.

Example 4.1. Let $g_{T N}$ be the curve of AH (or EAdS) Taub-NUT metrics on the 4-ball $B^{4}$, cf. [38] for instance, given by

$$
g_{T N}=l^{2} E\left[\frac{\left(r^{2}-1\right)}{F(r)} d r^{2}+\frac{F(r)}{\left(r^{2}-1\right)} \theta_{1}^{2}+\frac{\left(r^{2}-1\right)}{4} g_{S^{2}(1)}\right],
$$

where $E \in(0, \infty)$ is any constant, $r \geq 1$, and

$F(r)=E r^{4}+(4-6 E) r^{2}+(8 E-8) r+4-3 E=(r-1)^{2}\left\{E(r+1)^{2}+4(1-E)\right\}>0$.

Here $\theta_{1} \in[0,2 \pi]$ parametrizes the circle $S^{1}$ in the Hopf fibration $S^{3} \rightarrow S^{2}$. The nut charge description of $g_{T N}$, cf. [39] for instance, given by

$$
g_{T N}=V^{-1} d r^{2}+V \theta_{1}^{2}+\left(r^{2}-n^{2}\right) g_{S^{2}},
$$

where

$$
V(r)=\frac{\left(r^{2}+n^{2}\right)-2 m r+l^{-2}\left(r^{4}-6 n^{2} r^{2}-3 n^{4}\right)}{\left(r^{2}-n^{2}\right)},
$$

is equivalent to (4.1) under the substitution $r \rightarrow n r$, with $n^{2}=\frac{l^{2} E}{4}$, with mass parameter $m$ given by

$$
m=\frac{l}{2} E^{1 / 2}(1-E)
$$

The AH Taub-NUT metric is self-dual Einstein and has conformal infinity $\gamma$ given by the "Berger sphere" with $S^{1}$ fibers of length $\beta=2 \pi E^{1 / 2} \in(0, \infty)$ over $S^{2}\left(\frac{1}{2}\right)$. The scalar curvature $R_{\gamma}$ of the boundary metric $\gamma$ satisfies

$$
R=8-2 E .
$$

Clearly $\gamma$ is real-analytic, as is the geodesic compactification with boundary metric $\gamma$. Since $g$ is self-dual, [40] implies that the $g_{(3)}$ term in the expansion 
(2.6), (see also (5.3) below), is given by

$$
g_{(3)}=\frac{1}{3} * d R i c,
$$

wher $R i c=R i c_{\gamma}$ is viewed as a vector valued 1 -form, $d$ is the exterior derivative and $*$ the Hodge $*$ operator, all with respect to $\gamma$. When $E=1$, $g_{T N}$ is the Poincaré metric.

The de Sitter continuation of $g_{T N}$ at $\mathcal{I}^{+}$is the dS Taub-NUT metric on $\mathbb{R} \times S^{3}$, cf. [41] for instance, given by

$$
g^{T N}=l^{2} E\left[-\frac{\left(\tau^{2}+1\right)}{A(\tau)} d \tau^{2}+\frac{A(\tau)}{\left(\tau^{2}+1\right)} \theta_{1}^{2}+\frac{\left(\tau^{2}+1\right)}{4} g_{S^{2}}\right],
$$

where $\tau \in(-\infty, \infty)$ and

$$
A(\tau)=E \tau^{4}-(4-6 E) \tau^{2}-(8 E-8) \tau+4-3 E .
$$

Again when $E=1, g^{d S}$ is the (exact) de Sitter metric. Changing $n \rightarrow i n$, $r \rightarrow i \tau$ transforms (4.3) into the nut form of $g^{T N}$.

The dS Taub-NUT metric $g^{T N}$ is complete and globally hyperbolic, without singularities, exactly when $A(\tau)>0$, for all $\tau$. A straightforward but lengthy calculation shows this is the case if and only if

$$
E \in\left[\frac{2}{3}, \frac{1}{3}(2+\sqrt{3})\right]
$$

For $E$ in the range (4.8), there are no closed time-like curves, in contrast to the AdS (or $\Lambda=0$ ) Taub-NUT metric, which always have such curves. (Friedrich's theorem [12] also guarantees the existence of some interval $\mid E-$ $1 \mid<\varepsilon$ which has the same overall global structure of pure de Sitter space).

Consider first the situation $E \in\left(\frac{2}{3}, \frac{1}{3}(2+\sqrt{3})\right)$. Then both $\mathcal{I}^{+}$and $\mathcal{I}^{-}$ are well-defined and $g^{T N}=g^{T N}(E) \in d S^{ \pm}$. Observe from the explicit form of (4.6) that

$$
\gamma^{-}=\gamma^{+} \text {and } g_{(3)}^{+}=-g_{(3)}^{-} ;
$$

here $g_{(3)}^{+}$is taken with respect to the past timelike direction $\partial_{\rho}$, while $g_{(3)}^{-}$ is taken with respect to the future timelike direction $\partial_{\rho}$, Thus, even though $g^{T N}$ is not time-symmetric when $E \neq 1$, there is no gravitational scattering from past to future conformal infinity; from (3.3), one has

$$
\mathcal{S}=i d,
$$

on the Taub-NUT curve. The AH continuation of the dS Taub-NUT metric at $\mathcal{I}^{-}$is the anti self-dual AH Taub-NUT metric (4.3), (due to the change in orientation), with $n$ replaced by $-n$, changing $m$ to $-m$; this metric has an isolated cone-like singularity at the origin $\{r=n\}$. 
Next consider the extreme values $E_{-}=\frac{2}{3}$ and $E_{+}=\frac{1}{3}(2+\sqrt{3})$, and let $\tau_{-}=-1, \tau_{+}=(2-\sqrt{3})$. At $E=E_{-}, A(\tau) \geq 0$ for all $\tau$, with $A(\tau)=0$ only at $\tau_{-}$. Moreover, $\tau_{-}$is a degenerate zero in that $A^{\prime}\left(\tau_{-}\right)=$ 0 . Exactly the same remarks hold at $E_{+}$and $\tau_{+}$. At the extreme values $E_{ \pm}$, the metric $g^{T N}=g^{T N}\left(E_{ \pm}\right)$is still geodesically complete and globally hyperbolic. However, neither of these two metrics is in $d S^{ \pm}$. At $E_{-}$for instance, there are two solutions $g_{p}^{T N}$ and $g_{f}^{T N}$, parametrized by $\left(-\infty, \tau_{-}\right)$ and $\left(\tau_{-}, \infty\right)$ respectively, each complete and globally hyperbolic, with $g_{p}^{T N} \in$ $d S^{-}, g_{f}^{T N} \in d S^{+}$, with same conformal infinities $\gamma^{+}=\gamma^{-}=\gamma\left(E_{-}\right)$on each end. This situation corresponds of course to Case I in Theorem 3.1.

As one goes to future infinity in $g_{p}^{T N}$, or past infinity in $g_{f}^{T N}$, the spatial metric degenerates, by collapsing the $S^{1}$ fiber in the Hopf fibration to a point, while the radius of the base $S^{2}$ converges to $\left(E_{-}\left(\tau_{-}^{2}+1\right) / 4\right)^{1 / 2}$. The curvature of these metrics is uniformly bounded. Exactly the same structure holds at $E_{+}$.

The two extreme metrics $g_{p}^{T N}$ and $g_{f}^{T N}$ are analogous to extreme black hole solutions. For $E$ outside the range (4.8), $A(\tau)$ acquires up to 4 zeros, so as $\tau$ ranges over all $\mathbb{R}$, one has several copies of the Taub-NUT metrics glued along horizons by analytic continuation; cf. [19] for a detailed description.

One expects that exactly the same behavior holds for all dS Bianchi IX metrics, and it would be interesting to see if this is the case. The corresponding AdS Bianchi IX metrics are also self-dual, and have been described in detail in [30].

There are Taub-NUT metrics in higher (even) dimensions, cf. [19], [42], and the discussion above holds in essentially the same form.

As a final remark, as $E \rightarrow \infty$, the AH Taub-NUT metrics limit on the Bergmann or complex hyperbolic metric on the unit ball in $\mathbb{C}^{2}$; the corresponding conformal infinity is degenerate, cf. [43] for example. This limit corresponds to taking $R_{\gamma} \rightarrow-\infty$, and hence by (3.2), there is no dS continuation of the Bergmann metric.

Examples 4.2. The AH Schwarzschild metrics are a curve of metrics on $M=\mathbb{R}^{2} \times S^{n-1}$ given by

$$
g_{S c h}=V^{-1} d r^{2}+V d \theta^{2}+\left(\frac{r}{l}\right)^{2} g_{S^{n-1}(l)},
$$

where

$$
V(r)=1+\left(\frac{r}{l}\right)^{2}-\frac{\mu}{r^{n-2}},
$$


Here $r \in\left[r_{+}, \infty\right)$, where $r_{+}$is the largest root of $V$, and the circular parameter $\theta \in[0, \beta], \beta=4 \pi l^{2} r_{+} /\left(n r_{+}^{2}+(n-2) l^{2}\right)$, with $\mu=2 m G$. It is easy to see that the conformal infinity of $g_{S c h}(m)$ is given by the conformal class of the product metric $\gamma$ on $S^{1}(\beta) \times S^{n-1}(l)$. As a function of $m \in(0, \infty)$, observe that $\beta$ has a maximum value of $\beta_{0}=4 \pi l^{2} /\left(1+l^{2}\right)(n(n-2))^{1 / 2}$, and for every $m \neq m_{0}$, there are two values $m^{ \pm}$of $m$ giving the same value of $\beta$. Thus two distinct metrics have the same conformal infinity.

The de Sitter continuation of the AH Schwarzschild metric is not exactly the dS Schwarzschild metric. Suppose first $n=3$. Then the continuation (at $\mathcal{I}^{+}$) is the dS Kantowski-Sachs metric on $\mathbb{R} \times S^{1} \times S^{2}$, given by

$$
g^{K S}=-V^{-1} d t^{2}+\alpha^{2} V d \theta^{2}+t^{2} g_{S^{2}(l)},
$$

where

$$
V=V(t)=t^{2}-1+\frac{\mu}{t}
$$

and we have set $l=1$. Here $\alpha>0$ is a free parameter; a continuous continuation from $g_{S c h}$ to $g^{K S}$ then requires $\alpha=1$ but $g^{K S}$ is defined for any $\alpha>0$; see [44] for a general discussion of these metrics.

By the result of Andersson-Galloway [33] discussed in $\S 3, \mathcal{I}^{-}=\emptyset$ for these metrics, i.e. for the maximal globally hyperbolic developments. To see this in detail, consider first the case $\mu=0$; note however that the $\mathrm{AH}$ Schwarzschild metric $g_{S c h}$ with $\mu=0$ is degenerate, i.e. not defined. The metric $g^{K S}(0)$ is a quotient of a domain in pure de Sitter space by a discrete $\mathbb{Z}$-action. The Penrose diagram for pure de Sitter space is a square with the $t$-level curves in (4.12) given by hyperbolas in the upper triangle when $t>1$; for $t=1$, the level curves are the diagonals of the square. The $\mathbb{Z}$-action is a boost symmetry (of length determined by $\alpha \cdot \beta$ ). Near the horizon $t=1$, the geometry is that of a round 2-sphere $t^{2} g_{S^{2}(1)}$ times the Misner spacetime, cf. [8]. Thus, there are analytic continuations across the horizon, giving rise to closed time-like curves. When $\mu>0$, these closed time-like curves persist, but there is now in addition a singularity at $t=0$. In both cases, the maximal globally hyperbolic spacetime has $\mathcal{I}^{-}=\emptyset$.

The universal cover of the dS Kantowski-Sachs solutions are the dS Schwarzschild metrics; in terms of (4.12), one just replaces the circular parameter $\theta \in[0, \beta]$ by $r \in \mathbb{R}$; then (4.12) becomes the usual form of the dS Schwarzschild metric in the exterior of the cosmological horizon. When $\mu=0$, this gives the pure de Sitter metric outside the horizon, while the extension inside the horizon is de Sitter in static coordinates, given by, (interchanging $t$ and $r$ coordinates),

$$
g_{d S}=-\left(1-r^{2}\right) d t^{2}+\left(1-r^{2}\right)^{-1} d r^{2}+r^{2} g_{S^{2}(1)} .
$$


Future conformal infinity $\mathcal{I}^{+}$has thus changed from the compact manifold $S^{1} \times S^{2}$ to the universal cover $\mathbb{R} \times S^{2}$. Of course, this can be conformally changed to $S^{3} \backslash\{q \cup-q\}$, where $q$ and $-q$ are two antipodal points, and so extended to $S^{3}$, giving rise to the usual compactification of pure de Sitter in global coordinates. The translation Killing field, (the analytic continuation of the static time-like Killing field $\partial_{t}$ in (4.14)), then becomes a conformal Killing field, (the dilatation field) on $S^{3}$.

When $\mu>0$, one again has $\mathcal{I}^{+}=\mathbb{R} \times S^{2}$. However, the singularity at $t=0$ in (4.12) now propagates to future infinity, giving a 2 -sphere of 0 radius times $\mathbb{R}$ at $\mathcal{I}^{+}$. Analytic continuation through horizons gives the usual infinite Penrose diagram for the maximally extended dS Schwarzschild metrics, cf. [1].

On the AH side, $\mathbb{R}^{2} \times S^{2}$ is simply connected, so the $S^{1}$ at infinity cannot be unwrapped. Consider however the AdS Schwarzschild metric obtained from the AH Schwarzschild metric (4.10) by replacing $\theta$ by it;

$$
g_{S c h}=-V d t^{2}+V^{-1} d r^{2}+r^{2} g_{S^{2}(1)} .
$$

This static metric has conformal infinity of the form $\mathcal{I}^{+}=\mathbb{R} \times S^{2}$, so its continuation across $\mathcal{I}^{+}$is the dS Schwarzschild metric.

Now consider the same situation with $n>3$. The continuation of an AH Schwarzschild metric is again a Kantowski-Sachs metric of the form (4.12), (with $S^{n-1}(1)$ in place of $S^{2}(1)$ ), and with $V$ of the form

$$
V=V(t)=t^{2}-1+\frac{\mu}{t^{n-2}} .
$$

The terms $\mu / r^{n-2}$ in (4.11) and $\mu / t^{n-2}$ in (4.16) essentially determine the $g_{(n)}$ term in the Fefferman-Graham-Starobinsky expansion, or more precisely its deviation from the $g_{(n)}$ term for pure AH or dS space. The relation (2.17) of the $g_{(n)}$ terms in the $\mathrm{AH}$ and $\mathrm{dS}$ expansions then requires that

$$
\mu_{d S}= \pm \mu_{A H}
$$

where + occurs if $n \equiv 2,3(\bmod 4)$ and - occurs if $n \equiv 0,1(\bmod 4)$. Thus, the masses $\mu$ agree, and are both positive, only when $n \equiv 2,3(\bmod 4)$. When $n=4$ for example, the positive mass AH Schwarzschild metric continues to the negative mass Kantowski-Sacks metric and vice versa. These negative mass metrics of course have (naked) curvature singularities; cf. $\S 5$ for further discussion.

The discussion above generalizes easily to higher genus black holes, by replacing the constant 1 by $k, k=0$ or -1 , and $S^{2}$ with the torus $T^{2}$ or a surface of higher genus $\Sigma_{g}$ respectively. Moreover, these spaces may be 
replaced by Einstein metrics on any $(n-1)$-dimensional manifold, cf. [39], [45].

\section{$5 \quad$ Holographic Renormalization.}

Einstein metrics (2.2) are critical points of the Einstein-Hilbert action. For Riemannian (i.e Euclidean) metrics, the action is usually taken to be

$$
I=-\frac{1}{16 \pi G} \int_{M}(R-2 \Lambda) d v-\frac{1}{8 \pi G} \int_{\partial M} H d A,
$$

where $H$ is the mean curvature of the boundary. (The sign conventions are based on path integral considerations, cf. [46]). However, both terms in (5.1) are infinite on AH Einstein metrics. The main idea in the method of holographic renormalization is that one may find natural counterterms, depending only on the intrinsic geometry of the boundary metric $\gamma$, (or the metric on the cutoff at $t=\varepsilon$ ), such that if $I$ is renormalized by subtraction of these counterterms, the renormalized action $I^{\text {ren }}$ is finite, cf. [13], [14]. (Very briefly, the counterterms are obtained from the expansion of the action determined by (2.6) or (2.8)). If $n$ is odd, $I^{r e n}$ depends only on $(M, g)$, and not on any particular choice of compactification. However, if $n$ is even, $I^{r e n}$ does depend on the compactification, i.e. on boundary metric $\gamma$, and not just on the conformal class $[\gamma]$. This behavior is closely related to the absence or presence of the conformal anomaly in odd and even dimensions respectively.

The variation of $I^{r e n}$ at a given $\mathrm{AH}$ Einstein metric $g$ is given by

$$
d I^{r e n}(h)=\frac{d}{d t} I^{r e n}(g+t h),
$$

where $h$ is tangent to the space $E$ of AH Einstein metrics; (this is a smooth manifold, cf. [25]). The differential $d I^{r e n}$ is the holographic stress-energy tensor; it is a 1-form on $E$ and corresponds to the renormalization of the quasi-local Brown-York stress-energy [47]. Via the AdS/CFT correspondence, $T=2 d I^{r e n}$ gives the expectation value of the stress-energy tensor of the CFT on $\partial M$, in all known cases.

Since Einstein metrics are critical points of $I$ or $I^{r e n}$, it is clear that $d I^{r e n}$ must be supported on $\partial M$. If $n$ is odd, it is proved in [14], cf. also [40], that

$$
T=2 d I^{r e n}=-\frac{n}{16 \pi G} g_{(n)},
$$

while if $n$ is even,

$$
T=2 d I^{r e n}=-\frac{n}{16 \pi G}\left(g_{(n)}+r_{(n)}\right),
$$


where $r_{(n)}$ is explicitly determined by $\gamma$ and covariant derivatives of its curvature; it depends only on the lower order terms in the FG expansion. Thus

$$
\langle T, h\rangle=-\frac{n}{16 \pi G} \int_{\partial M}\left\langle g_{(n)}+r_{(n)}, h_{(0)}\right\rangle d v_{\gamma},
$$

where $h_{(0)}$ is the variation of the boundary metric $\gamma$ induced by $h$. The complexity of the term $r_{(n)}$ grows rapidly in $n$; explicit expressions for $n=$ $2,4,6$ are given in [14]. (The sign in (5.3) or (5.4) differs from that in [14], but agrees with the signs in [13], [39], [40]. With this choice of sign, the mass of pure $\mathrm{AH}_{5}=\mathrm{EAdS}_{5}$ is positive in static coordinates $\left.\left(\partial M=S^{1} \times S^{3}\right)\right)$.

For Lorentzian metrics, the action has the form

$$
I=\frac{1}{16 \pi G} \int_{M}(R-2 \Lambda) d v+\frac{1}{8 \pi G} \int_{\partial M} H d A .
$$

Note that this has the opposite sign to (5.1). For general AdS metrics, this action cannot be renormalized to a finite expression as in the $\mathrm{AH}$ case, since such metrics are not conformally compact, and in general are time dependent, with $t \in \mathbb{R}$. The action can be renormalized to a finite value however for stationary AdS metrics.

In any case, for general AdS metrics one does have a renormalized stressenergy tensor $T_{\mu \nu}$. This is again given by (5.3), (5.4) but with the opposite sign. The stress-energy tensors $T^{A d S}$ of a static AdS metric $(M, g), M=$ $\mathbb{R} \times \Sigma$, and the corresponding $T^{A H}$ of the $\mathrm{AH}$ metric $(M, g)$ obtained by setting $\theta=i t$ are then related by

$$
T_{t t}^{A d S}=T_{\theta \theta}^{A H}, \quad T_{\Sigma}^{A d S}=-T_{\Sigma}^{A H} .
$$

To illustrate on a concrete example, let $g_{S c h}^{A H}$ and $g_{S c h}^{A d S}$ be the $\mathrm{AH}$ and AdS Schwarzschild metrics respectively, with static compactification. Then the definitions above give

$$
T_{S c h}^{A H}=-\frac{1}{16 \pi G}\left(\frac{\mu}{l^{n-2}}+\frac{2 c_{n}}{n-1}\right)\left((1-n) d \theta^{2}+g_{S^{n-1}(1)}\right),
$$

while

$$
T_{S c h}^{A d S}=\frac{1}{16 \pi G}\left(\frac{\mu}{l^{n-2}}+\frac{2 c_{n}}{n-1}\right)\left(-(1-n) d t^{2}+g_{S^{n-1}(1)}\right),
$$

where $c_{n}=0$ if $n$ is odd and $c_{n}=(-1)^{n / 2} \frac{(n-1) ! !^{2}}{n !}$ if $n$ is even, cf. [39] and [45].

On the de Sitter side, since spatial infinity is compact, the action (5.6) can be holographically renormalized in exactly the same way as on the $\mathrm{AH}$ side, and the same renormalization procedure gives a holographic stress-energy 
tensor $T^{d S}=2 d I^{r e n}$. Because the $\mathrm{AH}$ action (5.1) and dS action (5.6) are both positive, one thus has

$$
T^{d S}\left(g^{d S}\right)=T^{A H}\left(g^{A H}\right),
$$

and $T^{d S}$ is given by (5.3) or (5.4), with the de Sitter terms $g_{(k)}^{d S}$ in place of the AH terms $g_{(k)}^{A H}$.

If now $g^{d S}$ is the analytic continuation of $g^{A H}$ in the sense of Theorem 2.1 , the formula (2.17) implies

(11) $T^{d S}=-T^{A H}$, if $n \equiv 3(\bmod 4)$, and $T^{d S}=T^{A H}$, if $n \equiv 1(\bmod 4)$.

Similarly,

$$
T^{d S}=T^{A H} \text {, if } n=4, \text { and } T^{d S}=-T^{A H} \text {, if } n=2 .
$$

Curiously, it is not clear if analogous formulas hold in general for $n$ even, $n \geq 6$, since the term $r_{(n)}$ does not seem to have a definite sign change under the correspondence (2.18) in this range, according to [14].

We now turn to a discussion of conserved quantities of AH and dS metrics associated with conformal Killing fields at conformal infinity, following [13][15]. First, it should be noted that a generic conformal infinity $(\partial M,[\gamma])$ has no conformal Killing fields, and so the discussion applies at the outset only to a restricted class of spaces.

We begin in the $\mathrm{AH}$ setting. Let $(M, g)$ be an $\mathrm{AH}$ Einstein metric with conformal infinity $(\partial M, \gamma)$. One may define a conserved quantity associated with any conformal Killing field $K$ on $(\partial M, \gamma)$ by

$$
Q=\int_{S}\langle T(K), \nu\rangle
$$

where $T$ is the holographic stress-energy tensor, $S$ is a slice to the orbits of the Killing field on $(\partial M, \gamma)$, and $\nu$ is the unit normal to $S$, in the direction $K$. The integral (5.13) is independent of $S$, since $T$ is transverse-traceless. If $n$ is odd, $Q$ depends only on the Einstein metric $(M, g)$ and choice of Killing field $K$ on $(\partial M, \gamma)$. However, if $n$ is even, $Q$ depends on the choice of boundary metric $\gamma \in[\gamma]$.

As mentioned in $\S 2$, any conformal Killing field at conformal infinity extends to a Killing field of any (globally smooth) AH Einstein bulk metric $(M, g)$. Furthermore, at least in many circumstances, if $K$ is static, i.e. hypersurface orthogonal on $\partial M$, then $K$ is also static on $(M, g)$, cf. [48].

The same definition (5.13) holds for AdS metrics $(M, g)$. In particular, if $(M, g)$ is stationary in a neighborhood of infinity, then the (holographically 
renormalized) mass of $(M, g)$ is given by

$$
m=\int_{S}\langle T(K), \nu\rangle,
$$

where $S$ is a spacelike slice for the Lorentz metric $\gamma$ on $\partial M$ and $K$ is the timelike Killing field. Again, the value of $m$ depends on the choice of boundary metric $\gamma$ in $[\gamma]$ when $n$ is even.

In particular, if the AdS metric $(M, g)$ is globally static (near $\partial M$ ), then $(M, g)$ can be Wick rotated to an AH Einstein metric, $(t \rightarrow i t=\theta)$. The $\mathrm{AH}$ mass and the AdS mass are then equal by (5.7). While in general stationary AdS metrics cannot be Wick rotated to stationary AH metrics, this can be done in many interesting concrete cases, such as the AdS Taub-NUT and Kerr metrics. In such a situation, the masses again agree by (5.7).

Conserved quantities can be defined in same way as (5.13) for metrics $(M, g)$ in $d S^{+}$or $d S^{-}$having Killing fields at $\mathcal{I}^{+}$or $\mathcal{I}^{-}$. Since $\mathcal{I}$ is spacelike, it is not immediately clear which of these quantities should correspond to mass, and which to angular momentum-type quantities. A natural proposal due to Balasubramanian, de Boer and Minic [15], is that $Q$ in (5.13) gives the mass of $(M, g)$ at $\mathcal{I}^{+}$when the Killing field is the "analytic continuation" of a timelike vector field of $(M, g)$, which is asymptotically Killing and static near $\mathcal{I}^{+}$. In more detail, since the metric (locally) asympotically approaches the de Sitter geometry, one can write the metric near $\mathcal{I}^{+}$in an approximately static chart, as in (4.14), and then continue the approximate timelike Killing field into the exterior region of the cosmological horizon to obtain an approximate spacelike Killing field. When this process leads to a Killing field on $\mathcal{I}^{+}$, then the associated conserved quantity is defined to be the mass. One difficulty in general is that if the data $\left(g_{(0)}, g_{(n)}\right)$ at $\mathcal{I}^{+}$are not analytic, it is not clear how to actually carry out an analytic continuation; there may be no coordinates in which the metric is analytic.

Consider first the case of pure de Sitter spacetime. The exterior of the static chart leads to $\left(\mathcal{I}^{+}, \gamma\right)$ given by the round product metric on $\mathbb{R} \times$ $S^{n-1}(1)$, with Killing field given by translation along the $\mathbb{R}$-direction. In general, if $K=\partial_{s}$ is a non-vanishing conformal Killing field on $\mathcal{I}^{+}$, then locally the topology of $\mathcal{I}^{+}$is $I \times S$, with metric of the form

$$
\gamma=e^{\phi s}\left[N^{2} d s^{2}+h_{i j}\left(d x_{i}+n_{i} d s\right)\left(d x_{j}+n_{j} d s\right)\right],
$$

where $h$ is a metric on $S, N, n_{i}$ and $h_{i j}$ are independent of $s$ and $\phi: S \rightarrow \mathbb{R}$.

Suppose that $K$ is static, so that $n_{i}=0$. Then $\mathcal{I}^{+}$is topologically of the form $\mathbb{R} \times S$, and the representation (5.15) holds globally over all $\mathbb{R}$. The continuation of $(M, g) \in d S^{+}$across $\mathcal{I}^{+}$gives an AH Einstein metric, with 
static Killing field $K$, defined at least in a neighborhood of conformal infinity $\partial M=\mathcal{I}^{+}$. By Wick rotation, this is equivalent to a static AdS Einstein metric, for which one has the natural mass definition given by (5.14). Thus, $K$ on $\mathcal{I}^{+}$is time-like with respect to the naturally associated AdS metric. This notion of mass agrees with the BBM mass [15] discussed above.

Suppose next the Killing field $K$ on $\mathcal{I}^{+}$is stationary and not static. Then the metric $\gamma$ on $\mathcal{I}^{+}$either has the global form (5.15) on $\mathbb{R} \times S$, or $\mathcal{I}^{+}$is a non-trivial $S^{1}$ bundle over $S$, with the fiber $S^{1}$ trivial, or of finite order, in $\pi_{1}\left(\mathcal{I}^{+}\right)$. In the latter case, one cannot unwrap the orbits of the Killing field to $\mathbb{R}$ by passing to a covering space. The AH Einstein continuation will also be stationary, and as mentioned above, in certain circumstances this can again be Wick rotated to a stationary AdS metric having the same mass. Note that in general the resulting AdS metric then has closed timelike curves at conformal infinity. For example, the dS Taub-NUT metric continues to the AH Taub-NUT metric which can be rotated to the AdS Taub-NUT metric.

The following result relates the mass of these two metrics.

Proposition 5.1. Let $m_{d S}$ be the holographic mass of the de Sitter-type metric $(M, g) \in d S^{+}$, with static or stationary Killing field $K$ on $\mathcal{I}^{+}$, and let $m_{A d S}$ be the mass of the associated AdS metric $(M, g)$. If $n$ is odd, then

(16) $m_{d S}=-m_{A d S}$, if $n \equiv 3(\bmod 4)$, and $m_{d S}=m_{A d S}$, if $n \equiv 1(\bmod 4)$. Also,

$$
m_{d S}=m_{A d S}, \text { if } n=2, \text { and } m_{d S}=-m_{A d S} \text {, if } n=4 .
$$

Proof: This follows immediately from the discussion above, together with (5.11)-(5.12).

We use these results to discuss the maximal mass conjecture proposed in [15], that if $(M, g) \in d S^{+}$has mass greater than the mass of pure de Sitter space, then $M$ has a cosmological singularity. A reasonable definition here is that $(M, g) \in d S^{+}$has a cosmological singularity if either $(M, g)$ contains a naked singularity, (as for instance the negative mass dS Schwarzschild metric), or

$$
\mathcal{I}^{-}=\emptyset,
$$

i.e. there is no partial conformal completion at past infinity for the globally hyperbolic spacetime $(M, g)$. As a very special case, the conjecture implies that among all spaces in $d S^{ \pm}$, the mass of the pure de Sitter space is maximal. In particular, in dimension 4 ,

$$
m_{d S^{ \pm}} \leq 0=m_{0}
$$


where $m_{0}$ is the mass of pure de Sitter. This conjecture has been verified in a number of cases [15], [17]; see also [18]-[20] for a discussion of possible counterexamples.

Consider the family of dS Taub-NUT metrics $g^{T N}$ analysed in $\S 4$. For $E$ in the range (4.8), $g^{T N} \in d S^{ \pm}$. On the other hand, (4.4), (4.5), (5.3) and (5.16) show that the mass of $g^{T N}$ is given by

$$
m\left(g^{T N}\right)=-m\left(g_{T N}\right)=c \frac{l}{2} E^{1 / 2}(E-1),
$$

for a fixed numerical constant $c$. Hence, when $E>1$, the mass is greater than that of pure de Sitter.

One might consider strengthening the conjecture to require that $\gamma^{+}$is the round metric $\gamma_{0}$ on $S^{3}$, so that $g$ is strongly $d S^{+}$. The conformal Killing field giving the mass is thus fixed to be the dilatation field (with south pole to north pole flow), as are all the quantities in (5.13)-(5.14) except $T$. However, as discussed in $\S 3$, Friedrich's theorem [12] implies that the data $\left(\gamma_{0}, g_{(3)}\right)$ determine a unique solution $(M, g) \in d S^{ \pm}$, for any sufficiently small transverse-traceless form $g_{(3)}$. Since $T \sim g_{(3)}, T$ is freely specifiable as long as it is small, and so from (5.14) it is clear that there exists $\varepsilon_{0}>0$ such that $m$ may assume any value in

$$
m \in\left(-\varepsilon_{0}, \varepsilon_{0}\right),
$$

within the class of spaces in $d S^{ \pm}$. We recall also that in dimension 4 , the mass is independent of the choice of representative $\gamma \in[\gamma]$, so that (5.21) also holds if $\mathcal{I}^{+}$is given by static coordinates, $\mathcal{I}^{+}=\mathbb{R} \times S^{2}$, with $\gamma_{0}$ the round product metric.

There are well-known positive mass theorems in AdS or AH spaces, cf. [49] for recent work and references. However, these require that $(M, g)$ is strongly $\mathrm{AH}$ in that the conformal infinity is the round product metric on $\mathbb{R} \times S^{n-1}$ or the round metric on $S^{n}$. The only globally smooth Einstein spaces with such conformal infinity are hyperbolic $n+1$-space, or $\operatorname{AdS}_{n+1}$ and so these results are uninteresting in this context. The proper context for the positive mass results is that $(M, g)$ is a strongly AH initial data set in a $\Lambda<0$ vacuum spacetime of one higher dimension, so that $(M, g, K)$ satisfies the constraint equations, not the Einstein equations. On the other hand, for any boundary metric $\gamma$ on $S^{3}$ or $S^{n}$ close to the round metric, there are global AH Einstein metrics with boundary metric $\gamma$, cf. [50], and for such spaces, the holographic mass will also satisfy (5.21).

In general even dimensions, the Taub-NUT metrics on $M=\mathbb{R} \times S^{n}, n$ odd, within $d S^{ \pm}$again have mass satisfying (5.21), and so also violate (5.19). Moreover, one also expects that Friedrich's theorem holds in all dimensions, 
especially in the case where $\left(\mathcal{I}^{+}, \gamma\right)=\left(S^{n}, \gamma_{0}\right)$, where $\gamma_{0}$ is the round metric on $S^{n}$ so that there are no log terms in the expansion, (the variation of the conformal anomaly vanishes). Note also that although the mass depends on the choice of representative $\gamma \in\left[\gamma_{0}\right]$ on $S^{n}$, the relations

$$
m>m_{0} \text {, or } m<m_{0},
$$

are independent of this choice; if either inequality holds for one choice of $\gamma \in\left[\gamma_{0}\right]$, then it holds for all choices in $\left[\gamma_{0}\right]$.

\section{Appendix}

In this Appendix, we carry out the computations verifying (2.17). The equation (2.5) states that

$$
\rho \ddot{g}_{\rho}-(n-1) \dot{g}_{\rho}-2 H g_{\rho}=\rho L,
$$

where $L=\left[2 R i c_{\rho}-H \dot{g}_{\rho}+\left(\dot{g}_{\rho}\right)^{2}\right]$. Also, the Gauss-Codazzi equations for $S(\rho) \subset(M, \bar{g})$ and the trace of the Riccati equation give

$$
\begin{gathered}
\operatorname{Ric}(T, X)=(\delta A+d H)(X)=0, \\
H^{\prime}+|A|^{2}+2 n R=0,
\end{gathered}
$$

where $R$ is the scalar curvature of $S(\rho)$. Equivalently, in local geodesic coordinates,

$$
\begin{gathered}
\rho \ddot{g}_{i j}-(n-1) \dot{g}_{i j}-g^{k l} \dot{g}_{k l} g_{i j}=\rho\left(2 R i c_{i j}-\frac{1}{2} g^{k l} g_{k l}^{\prime} g_{i j}^{\prime}+g^{k l} g_{i k}^{\prime} g_{j l}^{\prime}\right), \\
g^{k l}\left(\nabla_{l} \dot{g}_{i k}-\nabla_{i} \dot{g}_{k l}\right)=0 \\
\frac{1}{2} g^{k l} \dot{g}_{k l}+\frac{1}{4} g^{i k} \dot{g}_{i j} \dot{g}_{j k}+2 n R=0 .
\end{gathered}
$$

We need to divide into the cases $n$ odd or $n$ even.

Case I. $n$ odd.

Suppose first $n=3$. Differentiating (A.1) once gives

$$
\rho g_{\rho}^{(3)}-g_{\rho}^{(2)}-2\left(H g_{\rho}\right)^{(1)}=(\rho L)^{(1)} \text {. }
$$

Hence, at $\rho=0$,

$$
-g_{(2)}=H^{\prime}+R i c_{\gamma}=R i c_{\gamma}-\frac{R_{\gamma}}{4} \gamma .
$$

Differentiating again gives

$$
\rho g_{\rho}^{(4)}-2\left(H g_{\rho}\right)^{(2)}=(\rho L)^{(2)} .
$$

The term $R i c_{\rho}$ involves two tangential derivatives of $g_{\rho}$. Since $g^{(1)}=0$ at $\rho=0$, the right side of (A.6) vanishes at $\rho=0$. Further, since $\left(H g_{\rho}\right)^{(2)}=$ 
$\operatorname{ctrg}_{(3)} g$, (A.6) implies $\operatorname{trg}_{(3)}=0$. Here and below $c$ denotes a non-zero numerical constant, which may change from line to line. The Gauss-Codazzi equation (A.2) then gives $\delta g_{(3)}=0$. However, the transverse-traceless part of $g_{(3)}$ is undetermined by (A.6).

Differentiating further gives

$$
\rho g_{\rho}^{(5)}+g_{\rho}^{(4)}-2\left(H g_{\rho}\right)^{(3)}=(\rho L)^{(3)},
$$

so that at $\rho=0$,

$$
g_{(4)}-\operatorname{trg}_{(4)} g_{(0)}+\operatorname{ctrg}_{(2)} g_{(2)}=L^{(2)}
$$

This determines the coefficient $g_{(4)}$ in terms of $g_{(2)}$ and its 2nd tangential derivatives. Taking the next derivative at $\rho=0$ gives,

$$
2 g_{(5)}-\operatorname{trg}_{(5)} g_{(0)}=L^{(3)}
$$

so that $g_{(5)}$ depends on $g_{(3)}$ and its 2nd tangential derivatives. Finally, a further differentiation gives

$$
3 g_{(6)}-\operatorname{trg}_{(6)} g_{(0)}=L^{(4)}+\text { l.o.t., }
$$

where l.o.t. denotes lower order terms already determined. This determines the trace-free part of $g_{(6)}$ in terms of $g_{(4)}$ and its 2nd tangential derivatives. The trace of (A.9) is 0 . However, taking the $\rho$-derivative of (A.3) 4 times also shows that $\operatorname{trg}_{(6)}$ is determined by $g_{(4)}$ and its 2 nd tangential derivatives. Continuing this process inductively, one thus sees that the higher coefficients $g_{(k)}$ are uniquely determined and depend on the 2nd tangential derivatives of $g_{(k-2)}$.

Consider next the dS side. Here the equation (2.5) is replaced by (2.11), giving

$$
\rho \ddot{g}_{\rho}-(n-1) \dot{g}_{\rho}-2 H g_{\rho}=-\rho L,
$$

We assume now

$$
g_{(0)}^{d S}=g_{(0)}^{A H} .
$$

The same arguments as above then give the relation

$$
g_{(2)}^{d S}=-g_{(2)}^{A H} .
$$

As before $g_{(3)}^{d S}$ is transverse-traceless, but is otherwise undetermined, and so may be specified arbitrarily. Thus, we choose

$$
g_{(3)}^{d S}=-g_{(3)}^{A H} .
$$

It then follows from (A.10), (A.13) and formulas as in (A.7)-(A.9) that

$$
g_{(4)}^{d S}=g_{(4)}^{A H}, \quad g_{(5)}^{d S}=g_{(5)}^{A H},
$$


while

$$
g_{(6)}^{d S}=-g_{(6)}^{A H}, \quad g_{(7)}^{d S}=-g_{(7)}^{A H},
$$

and so on.

The same analysis and pattern as above holds for arbitrary $n$ odd, with $g_{(n)}$ in place of $g_{(3)}$. This verifies (2.17) when $n$ is odd.

Now suppose $n=4$. Differentiating (A.1) once gives

$$
\rho g_{\rho}^{(3)}-2 g_{\rho}^{(2)}-2\left(H g_{\rho}\right)^{(1)}=(\rho L)^{(1)},
$$

so that at $\rho=0$,

$$
-g_{(2)}=\frac{1}{2}\left(R i c_{\gamma}-\frac{R_{\gamma}}{6} \gamma\right)
$$

Differentiating again gives

$$
\rho g_{\rho}^{(4)}-g_{\rho}^{(3)}-2\left(H g_{\rho}\right)^{(2)}=(\rho L)^{(2)} .
$$

As above, the right side of (A.18) vanishes at $\rho=0$. Taking the trace of the left-side of (A.18) implies $\operatorname{tr} g_{(3)}=0$, and hence $g_{(3)}=0$. At the next level, one has

$$
\rho g_{\rho}^{(5)}-2\left(H g_{\rho}\right)^{(3)}=(\rho L)^{(3)} \text {. }
$$

Setting $\rho=0$, the left side is $\operatorname{ctr} g_{(4)}+$ l.o.t. and hence $\operatorname{trg} g_{(4)}$ is determined by 2 nd derivatives of $g_{(2)}$. Similarly $\delta g_{(4)}$ is determined via (A.3). However, the right side of (A.19) is not pure trace in general. To obtain a consistent expansion, one needs to introduce log terms in the expansion for $g_{\rho}$. Thus, set

$$
g_{\rho}=g_{(0)}+\rho^{2} g_{(2)}+\rho^{4} g_{(4)}+\rho^{4} \log \rho h_{(4)} .
$$

Since $\left(\rho^{4} \log \rho\right)^{(5)}=24 \rho^{-1}$, (A.19) gives

$$
24 h-2\left(H g_{\rho}\right)^{(3)}=(\rho L)^{(3)} .
$$

It follows that $\operatorname{trh}(4)=0$, and by (A.3), $\delta h_{(4)}=0$ also. The equation (A.21) thus determines the transverse-traceless part of $h$, in terms of the 2nd tangential derivatives of $g_{(2)}$. ting

However, the transverse-traceless part of $g_{(4)}$ is undetermined. Next, set-

$$
g_{\rho}=g_{(0)}+\rho^{2} g_{(2)}+\rho^{4} g_{(4)}+\rho^{4} \log \rho h_{(4)}+\rho^{5} g_{(5)},
$$

gives

$$
\rho g_{\rho}^{(6)}+g_{\rho}^{(5)}-2\left(H g_{\rho}\right)^{(4)}=(\rho L)^{(4)} .
$$


Observe that $\rho\left(\rho^{4} \log \rho\right)^{(6)}+\left(\rho^{4} \log \rho\right)^{(5)}=0$, leaving the term $g_{\rho}^{(5)}-\operatorname{ctr} g_{(5)} g_{(0)}+$ l.o.t. on the left equal to the right side of (A.23). But the right side vanishes, and hence $g_{(5)}=0$. Thus set

$$
g_{\rho}=g_{(0)}+\rho^{2} g_{(2)}+\rho^{4} g_{(4)}+\rho^{4} \log \rho h_{(4)}+\rho^{6} g_{(6)}+\rho^{6} \log \rho h_{(6)} .
$$

Differentiating again gives

$$
\rho g_{\rho}^{(7)}+2 g_{\rho}^{(6)}-2\left(H g_{\rho}\right)^{(5)}=(\rho L)^{(5)} .
$$

For the $\rho^{4} \log \rho h_{(4)}$ term, one has $\rho\left(\rho^{4} \log \rho\right)^{(7)}+2\left(\rho^{4} \log \rho\right)^{(6)}=0$, while for the $\rho^{6} g_{(6)}$ term $\rho\left(\rho^{6} \log \rho\right)^{(6)} h_{(6)}=c h_{(6)}+c \log \rho h_{(6)}$. Expanding out the right side of (A.25), one finds that $h_{(6)}$ and $g_{(6)}$ depend on two tangential derivatives of $g_{(4)}$ and $h_{(4)}$, and lower order terms.

Continuing in this way inductively shows that the expansion (2.8) is even in powers in $\rho$, with $(\log \rho)^{k}$ terms appearing at higher orders. Each coefficient $g_{(k)}$ and $h_{(k)}$ depends on two derivatives of the lower order terms $g_{(k-2)}$ and $h_{(k-2)}$.

The dS side when $n=4$ is analysed exactly as in the case $n=3$. Thus, (A.10) holds and assuming (A.11), (A.12) holds just as before. The odd coefficients vanish on both dS and AH sides. Setting

$$
g_{(4)}^{d S}=g_{(4)}^{A H},
$$

one finds

$$
g_{(2 k)}^{d S}=(-1)^{k} g_{(2 k)}^{A H}, \text { and } h_{(4)}^{d S}=h_{(4)}^{A H} .
$$

The same arguments and patterns hold for any even $n$, and give (2.17).

\section{References}

[1] G.W. Gibbons and S.W. Hawking, Cosmological event horizons, thermodynamics, and particle creation, Phys. Rev. D, 15, (1977), 2738 .

[2] S.W. Hawking and I.G. Moss, Phys. Lett. B. 110, (1982), 35.

[3] A. Strominger, The dS/CFT correspondence, JHEP 0110, (2001), 034, hep-th/0106113.

[4] M. Spradlin, A. Strominger and A. Volovich, Les Houches lectures on de Sitter space, hep-th/0110007.

[5] J. Maldacena, The large $N$ limit of superconformal field theories and supergravity, Adv. Theor. Math. Phys. 2, (1998), 231, hepth/9711200. 
[6] S.S. Gubser, I. R. Klebanov, and A.M. Polyakov, Gauge theory correlators from non-critical string theory, Phys. Lett. B 428, (1998), 405, hep-th/9802109.

[7] E. Witten, Anti-de Sitter space and holography, Adv. Theor. Math. Phys. 2, (1998), 253, [hep-th/9802150.

[8] S.W. Hawking and G.F.R. Ellis, The Large Scale Structure of Space-Time, Cambridge Univ. Press, Cambridge, (1973).

[9] P.O. Mazur and E. Mottola, Weyl cohomology and the effective action for conformal anomalies, Phys. Rev. D 64, (2001), 104022, hep-th/0106051.

[10] J. de Boer and S.N. Solodukhin, A holographic description of Minkowski space-time, Nucl. Phys. B, 665, (2003), 545-593, hepth/0303006.

[11] K. Skenderis, Lecture notes on holographic renormalization, Class. Quantum Grav. 19, (2002), 5849-5876, hep-th/0209067.

[12] H. Friedrich, On the existence of $n$-geodesically complete or future complete solutions of Einstein's field equations with smooth asymptotic structure, Comm. Math. Phys. 107, (1986), 587-609.

[13] V. Balasubramanian and P. Kraus, A stress tensor for anti-de Sitter gravity, Comm. Math. Phys. 208, (1999), 413, hep-th/9902121.

[14] S. de Haro, K. Skenderis and S.N. Solodukhin, Holographic reconstruction of spacetime and renormalization in the AdS/CFT correspondence, Comm. Math. Phys. 217, (2001), 595-622, hepth/0002230.

[15] V. Balsubramanian, J. de Boer and D. Minic, Mass, entropy and holography in asymptotically de Sitter spaces, Phys. Rev. D 65, (2002), 123508, hep-th/0110108.

[16] R. Bousso, The holographic principle, Rev. Mod. Phys., 74, (2002), 825-874, hep-th/0203101.

[17] R.G. Cai, Y.S. Myung and Y.Z. Zhang, Check of the mass bound conjecture in de Sitter space, Phys. Rev. D, 65, (2002), 084019, hep-th/0110234.

[18] R. Clarkson, A.M. Ghezelbash and R.B. Mann, Mass, action and entropy of Taub-Bolt-dS spacetimes, Phys. Rev. Lett., 91, (2003), 061301, hep-th/0304097.

[19] R. Clarkson, A.M. Ghezelbash and R.B. Mann, Entropic N-bound and maximal mass conjecture violations in four dimensional TaubBolt(NUT)-dS spacetimes, Nucl. Phys. B, 674, (2003), 329, hepth/0307059. 
[20] V. Balasubramanian, Accelerating universes and string theory, Class. Quantum Grav. 21, (2004), S1337-S1357, hep-th/0404075.

[21] C. Fefferman and C.R. Graham, Conformal invariants, in: Élie Cartan et les Mathematiques d'Aujourd'hui, Astérisque, (1985), numero hors serie, Soc. Math. France, Paris, 95-116.

[22] C.R. Graham and K. Hirachi, The ambient obstruction tensor and Q-curvature, math.DG/0405068.

[23] C. Imbimbo, A. Schwimmer, S. Theisen and S. Yankielowicz, Diffeomorphisms and holographic anomalies, Class. Quantum Grav. 17, (2000), 1127-1138, hep-th/9910267.

[24] M. Anderson, Some results on the structure of conformally compact Einstein metrics, math.DG/0402198

[25] P. Chruściel, E. Delay, J.M. Lee and D.N. Skinner, Boundary regularity for conformally compact Einstein metrics, math.DG/0401386.

[26] A.A. Starobinsky, Isotropization of arbitrary cosmological expansion given an effective cosmological constant, JETP Lett, 37, (1983), 66-69.

[27] S. Kichenassamy, On a conjecture of Fefferman and Graham, Advances in Math. (2004), (in press).

[28] A. Rendall, Asymptotics of solutions of the Einstein equations with positive cosmological constant, gr-qc/0312020.

[29] M. Anderson, Einstein metrics with prescribed conformal infinity on 4-manifolds, math.DG/0105243.

[30] N. Hitchin, Twistor spaces, Einstein metrics and isomonodromic deformations, Jour. Diff. Geom. 42, (1995), 30-112.

[31] P. Chruściel, M. MacCallum and D. Singleton, Gravitational waves in general relativity XIV, Bondi expansions and the "polyhomogeneity" of $\mathcal{I}$, Proc. Roy. Soc. Lond, A436, (1992), 299-316, grqc/9305021.

[32] M. Anderson, Cheeger-Gromov theory and applications to general relativity, gr-qc/0208079.

[33] L. Andersson and G. Galloway, dS/CFT and spacetime topology, Adv. Theor. Math. Phys. 6, (2003), 307-327, hep-th/0202161.

[34] M. Anderson, Geometric aspects of the AdS/CFT correspondence, hep-th/0403087.

[35] G. Perelman, The entropy formula for the Ricci flow and its geometric applications, math.DG/0211159], Ricci flow with surgery on three-manifolds, math.DG/0303109. 
[36] S. Gao and R.M. Wald, Theorems on gravitational time delay and related issues, Class. Quant. Grav., 17, (2000), 4999-5008, gr-qc/0007021.

[37] F. Leblond, D. Marolf and R.C. Myers, Tall tales from de Sitter space I: renormalization group flows, JHEP, 0206, (2002), 052, hep-th/0202094.

[38] S.W. Hawking, C.J. Hunter and D.N. Page, Nut charge, antide Sitter and entropy, Phys. Rev. D 59, (1999), 044033, hepth/9809035.

[39] R. Emparan, C.V. Johnson and R.C. Myers, Surface terms as counterterms in the AdS/CFT correspondence, Phys. Rev. D 60, (1999), 104001, hep-th/9903238.

[40] M. Anderson, $L^{2}$ curvature and volume renormalization for AHE metrics on 4-manifolds, Math. Res. Lett, 8, (2001), 171-188, math.DG/0011051.

[41] D. Brill and F. Flaherty, Maximizing properties of extremal surfaces in General Relativity, Ann. Inst. Henri Poincaré, 28, (1978), 335-347.

[42] A.M. Awad and A. Chamblin, A bestiary of higher dimensional Taub-NUT AdS spacetimes, hep-th/0012240.

[43] H. Pedersen, Einstein metrics, spinning top motions and monopoles, Math. Annalen, 274, (1986), 35-59.

[44] P.V. Moniz, Kantowski-Sachs universes and the cosmic no-hair conjecture, Phys. Rev. D 47:10, (1993), 4315-4321.

[45] R. Emparan, AdS/CFT duals of topological black holes and the entropy of zero-energy states, JHEP 9906, (1999), 036, hepth/9906040.

[46] S.W. Hawking, The path integral approach to quantum gravity, in: General Relativity, an Einstein Centenary Survey, eds. S.W. Hawking and W. Israel, Cambridge Univ. Press, (1979).

[47] J. D. Brown and J.W. York, Jr., Quasilocal energy and conserved charges derived from the gravitational action, Phys. Rev. D 47:4, (1993), 1407-1419.

[48] M. Anderson, P. Chruściel and E. Delay, Nontrivial static, geodesically complete space-times with a negative cosmological constant II, $n \geq 5$, gr-qc/0401081.

[49] P. Chruściel and G. Nagy, The mass of asympototically anti-de Sitter space-times, Adv. Theor. Math. Phys. 5, (2002), 699-757.

[50] C. R. Graham and J. Lee, Einstein metrics with prescribed conformal infinity on the ball, Adv. Math. 87, (1991), 186-225. 